\title{
Tacit knowledge and the effect of pattern configuration on mental scanning
}

\author{
STEPHEN K. REED and HOWARD S. HOCK \\ Florida Atlantic University, Boca Raton, Florida 33431 \\ and \\ G. R. LOCKHEAD \\ Duke University, Durham, North Carolina 27706
}

\begin{abstract}
Two opposing hypotheses are that estimated distances may be used to predict scanning times in mental scanning experiments (Mitchell \& Richman, 1980; Pylyshyn, 1981) and that scanning times may be used to estimate distances on maps (Thorndyke, 1981). We tested these hypotheses by having people both estimate lengths and mentally scan diagonal lines, spirals, and mazes. There were large and consistent differences in the rate of scanning the three configurations, regardless of whether people scanned percepts or images. These differences could not be accounted for by differences in length estimates or by people's tacit knowledge of their scanning rates. We propose that people actually do mental scanning in a mental scanning task rather than use length estimates to generate scanning time and that they do not rely on scanning times to estimate length in a length estimation task.
\end{abstract}

The study of visual imagery has emerged as one of the central topics of cognitive psychology. Although there now appears to be extensive evidence that people use visual images to perform many cognitive tasks (Kosslyn \& Pomerantz, 1977), critics have occasionally questioned whether some of this evidence might have been produced by subjects who anticipated the results and generated appropriate data without using visual images. The tacit knowledge view, proposed by Pylyshyn (1981), suggests that subjects use their tacit knowledge of what should occur in reality to simulate operations on images without actually performing the operations.

A specific example is the Kosslyn, Ball, and Reiser (1978) finding that the time it takes to scan between two objects in a visual image is a linear function of the distance between the two objects. Kosslyn et al. interpret this finding as evidence that people can image a dot moving at a constant rate from one object to another. In contrast, the tacit knowledge view suggests that people could simulate this result by using their knowledge that it takes an object longer to move through a greater distance. The results might therefore be based on estimated time intervals rather than on actually imagining a dot move across an image (Pylyshyn, 1981, pp. 3334).

This alternative to image scanning was suggested previously by Mitchell and Richman (1980), who

We are grateful to Nelly Saavedra for testing subjects. Requests for reprints should be addressed to Stephen $\mathrm{K}$. Reed, Department of Psychology, Florida Atlantic University, Boca Raton, Florida 33431. observed that the Kosslyn et al. (1978) findings could have been produced by the demand characteristics of the experiment. If the hypothesized relationship between distance and scanning time was obvious to the subjects, they may have generated the expected results without actually scanning an image. In order to investigate subjects' tacit knowledge about the scanning task, Mitchell and Richman asked subjects to estimate how long it would take them to scan between two objects. The subjects produced a high correlation between distance and predicted scan time, demonstrating that they could anticipate Kosslyn et al.'s results.

The Mitchell and Richman (1980) findings support the plausibility of the tacit knowledge interpretation of visual scanning. If people can predict the relative time required to scan over different distances, they may respond on the basis of internally generated time intervals rather than on the basis of internally generated images. Neither Mitchell and Richman (1980) nor Pylyshyn (1981) argue that subjects' ability to predict the image-scanning data caused the original results. However, both correctly argue that if the image-scanning paradigm is to be a useful tool in the study of imagery, it is necessary to distinguish between the amount of variance due to the nature of imagery and the amount due to tacit knowledge and the demand characteristics of the experiment.

\section{Varying Pattern Configuration}

Although people can successfully predict that scanning time increases with distance (Mitchell \& Richman, 1980), they may not be able to predict how configura- 


\begin{tabular}{|c|c|c|c|c|c|c|c|c|}
\hline & 1 & 2 & 3 & 4 & 5 & 6 & 7 & 8 \\
\hline Line & ' & I & & & & & & \\
\hline Spiral & $c$ & c & อ & (C) & (e) & (C) & (2) & (C) \\
\hline Maze & $p$ & ש & 回 & @ & ㅁㅁ & 回 & 同 & \\
\hline
\end{tabular}

Figure 1. The three sets of line configurations.

tion influences scanning time. To examine this, we used a variation of the mental scanning task in which people scanned three different configurations. Figure 1 shows the three configurations, which are labeled line, spiral, and maze. If pattern configuration influences scanning time, but people are unaware of this effect, the data would support the view that people are actually scanning the patterns rather than using tacit knowledge and an internal clock to generate the results.

In investigating how configuration influences performance in scanning experiments, we are interested in two parameters. The first parameter is the slope of the best-fitting linear regression equation describing how scanning time increases as a function of length. This measures the rate of scanning. The second parameter is the $y$-intercept of that linear regression equation. This is particularly relevant when people are asked to estimate scanning time, because it may reflect tacit knowledge about configurations that is manifested by an additive constant that is independent of length. For example, people may have tacit knowledge that they will scan mazes more slowly than lines because they have to slow down for corners.

Estimating lengths. The tacit knowledge hypothesis proposes that people use their estimates of length to predict scanning time. In contrast to this, Thorndyke (1981) has proposed that scanning time is used to estimate length. His task required that subjects estimate distances on maps. Their distance estimates increased as a function of the number of points (such as the names of cities) along the route. To account for this finding, Thorndyke proposed that subjects perceptually scan a route or an image of a route and use scan duration to estimate path distance. The model assumes that intervening points slow down scanning, because subjects must stop and retrieve the name of the city to determine whether it is their destination point, and this results in estimates of greater distance. Thorndyke did not directly test this hypothesis by measuring scanning time.

The competing hypotheses, that distance estimates are used to predict scannning times (Mitchell \& Richman, 1980; Pylyshyn, 1981) and that scanning times are used to estimate distances (Thorndyke, 1981), suggest that we have an incomplete knowledge of how scanning time and distance are related. The following experiments investigate this relation by requiring that subjects participate in both a mental scanning and a length estimation task. In the first experiment, subjects were instructed to estimate the lengths of the 24 patterns shown in Figure 1 and to mentally scan the patterns so we could measure scanning time. In one condition, each pattern remained on the screen until the subject responded. This allowed for scanning of the pattern. In a second condition, the stimulus was on the screen for $.5 \mathrm{sec}$, which is not sufficient time to perform this task, and subjects were instructed to base their response on a visual image of the pattern. In the second experiment, subjects' tacit knowledge was examined by requiring that they estimate their scanning time for each pattern.

\section{EXPERIMENT 1}

\section{Method}

Subjects. The subjects were 24 undergraduates at Florida Atlantic University who received $\$ 3$ for participating. There were 12 subjects in the perception group and 12 subjects in the image group.

Stimuli. The stimuli consisted of the 24 patterns shown in Figure 1. They were rear-projected onto a 14 in. (height) $x$ 21 in. (width) translucent screen placed at eye height. The patterns appeared in the center of a rectangular slide frame that was $5.5 \mathrm{in}$. high and 8.625 in. long. The eight different lengths of each pattern were similar across patterns: $.8,1.7,2.8,3.5$, $4.3,5.1,5.9$, and 7.0 in. for the diagonal line; $.8,1.7,2.8,3.4$, $4.2,4.8,5.9$, and $6.8 \mathrm{in}$. for the spiral; and $.7,1.5,2.6,3.3,4.0$, $4.9,5.7$, and $6.7 \mathrm{in}$. for the maze. Subjects viewed the patterns from a distance of $3 \mathrm{ft}$. At this distance, the largest pattern in each series subtended a visual angle of $8 \mathrm{deg}$ for the diagonal line, $2.4 \mathrm{deg}$ for the spiral, and $1.6 \mathrm{deg}$ for the maze.

Procedure. Subjects in both the perception and image groups participated in two tasks: a visual scanning task and a length estimation task. For the perception condition, six of the subjects did the scanning task first and the other six did the length estimation task first. Each of the 24 patterns occurred twice during each task. The patterns occurred in a random order, except for the constraint that the second presentation of a pattern did not occur until all patterns had been presented.

Subjects read the following instructions for the visual scanning task: "This task was designed to study how well people can 
mentally scan objects. We will be showing you three different kinds of objects: diagonal lines, curved spirals, and straight spirals. The objects will appear, one at a time, on the screen in front of you. We want you to scan the object by imaging a little black speck moving from one end of the line to the other. Begin by focusing on the left end of the diagonal line (if a diagonal line is presented) or the end point inside a spiral. When you are ready to begin scanning, press the button on the switch and immediately image the speck moving along the line. The speck should move as quickly as possible while still remaining visible. As soon as you reach the end of the line, push the button again. The length of the line will vary for each of the objects. Try to move the speck at the same speed even though the line lengths and objects will change. You will have nine practice trials before we begin."

The following instructions were used for the length estimation task: "This task was designed to study how well people can estimate the lengths of lines. We will be showing you three different kinds of lines: diagonal lines, curved spirals, and straight spirals. The lines will appear, one at a time, on the screen in front of you. We want you to estimate the length of each line in inches. You may make your estimate to the nearest tenth of an inch if you wish. You will have nine practice trials before we begin."

The nine practice trials consisted of the shortest, the longest, and an intermediate length for each of the three patterns. Subjects did not receive any feedback during the practice trials but were encouraged to use a faster scanning rate if they exceeded $6 \mathrm{sec}$ on any of the practice patterns. After completing both tasks, subjects answered several questions about strategies.

For the image condition, the other 12 subjects followed the same procedure as the perception group, except that the patterns were presented for only $.5 \mathrm{sec}$ and the subjects were instructed to base their judgments on a visual image of the pattern. They were informed that the purpose of the experiment was to study how well people can scan visual images and use visual images to estimate the length of lines.

\section{Results}

Figure 2 shows the relation between estimated length and actual length for each of the three patterns (right column) and shows the scanning time for each pattern (left column), when the subjects scanned the pattern (top row), and when they scanned their image or memory of the pattern (middle row). Each point in these figures is the average of 24 judgments (12 subjects $X$ 2 judgments).

Length estimation. The group correlation between actual and estimated length was 1.00 for each of the three patterns in the perception condition, in which the pattern remained on the screen until the response. The average of the 12 individual subject correlations was .99 for the line, .97 for the spiral, and .98 for the maze. These results show that people are consistent in their length estimates and that this consistency is maintained for complex patterns, such as spirals and mazes, as well as for straight lines.

For the image condition, in which the pattern appeared for only $.5 \mathrm{sec}$, the correlations based on group averages were 1.00 for the line, .99 for the spiral, and 1.00 for the maze. The average of the individual correlations was .98 for the line, .97 for the spiral, and .96 for the maze. This extends the results of the perception condition by showing that people can maintain a high level of consistency in their length estimates even when they view a pattern for only a brief time.

The slopes of the regression lines in the perception condition were 1.03 for the line, .92 for the spiral, and 1.05 for the maze. The slopes in the image condition were .87 for the line, .69 for the spiral, and .75 for the maze. A 2 (groups) by 3 (patterns) analysis of variance revealed significant main effects for both group $[F(1,22)$ $=4.52, \mathrm{p}<.05]$ and pattern $[\mathrm{F}(2,44)=4.31, \mathrm{p}<.05]$, but the Group by Pattern interaction was not significant $[F(2,44)<1]$. The significant group effect was caused by the underestimation of length in the image condition. The underestimation is consistent with the results of Moyer, Bradley, Sorensen, Whiting, and Mansfield (1978), who reported that remembered size is less than perceived size when people judge length, area, or volume. ${ }^{1}$ The significant effect of pattern was caused by the underestimation of spiral length relative to line length. A Newman-Keuls analysis revealed that the slope for the spiral was significantly less $(p<.05)$ than the slope for the diagonal line, but neither pattern differed significantly from the maze. If people used length estimates to simulate mental scanning, scanning times for the spiral should be less than for the diagonal line. As shown in Figure 2, this did not occur.

Scan time. The group correlations between scan time and line length in the perception condition (top left panel in Figure 2) were .95 for the line, .99 for the spiral, and .99 for the maze. The average of the individual correlations was .86 for the line, .93 for the spiral, and .94 for the maze.

The close relation between scan time and physical length when the figure is scanned was maintained when subjects were asked to scan images (middle left panel in Figure 2). The group correlations were .99 for the line, .97 for the spiral, and .99 for the maze. The average of the individual correlations was .81 for the line, .91 for the spiral, and .94 for the maze.

Perhaps the most striking characteristic of the results was the effect of pattern on scan rate. As measured by the slopes of the regression lines, the rate of scanning in the perception condition was $.17 \mathrm{sec} / \mathrm{in}$. for the line, $.30 \mathrm{sec} / \mathrm{in}$. for the spiral, and $.44 \mathrm{sec} / \mathrm{in}$. for the maze. The corresponding values were $.23, .37$, and .47 for the image condition. A 2 (groups) by 3 (patterns) analysis of variance indicated significantly different scan rates across patterns $[F(2,44)=46.14, p<.01]$. All three scan rates differed significantly $(p<.01)$ from each other according to a Newman-Keuls analysis. Neither the effect of group $[F(1,22)<1]$ nor the Group by Pattern interaction $[F(2,44)<1]$ was significant. Scanning rates were essentially the same when subjects scanned a perceptual pattern as when they scanned an image of the pattern.

Table 1 summarizes the correlations and regression parameters found in Experiment 1. The table also shows the results of an experiment (labeled Experiment 1A) that we conducted before Experiment 1. Since the 

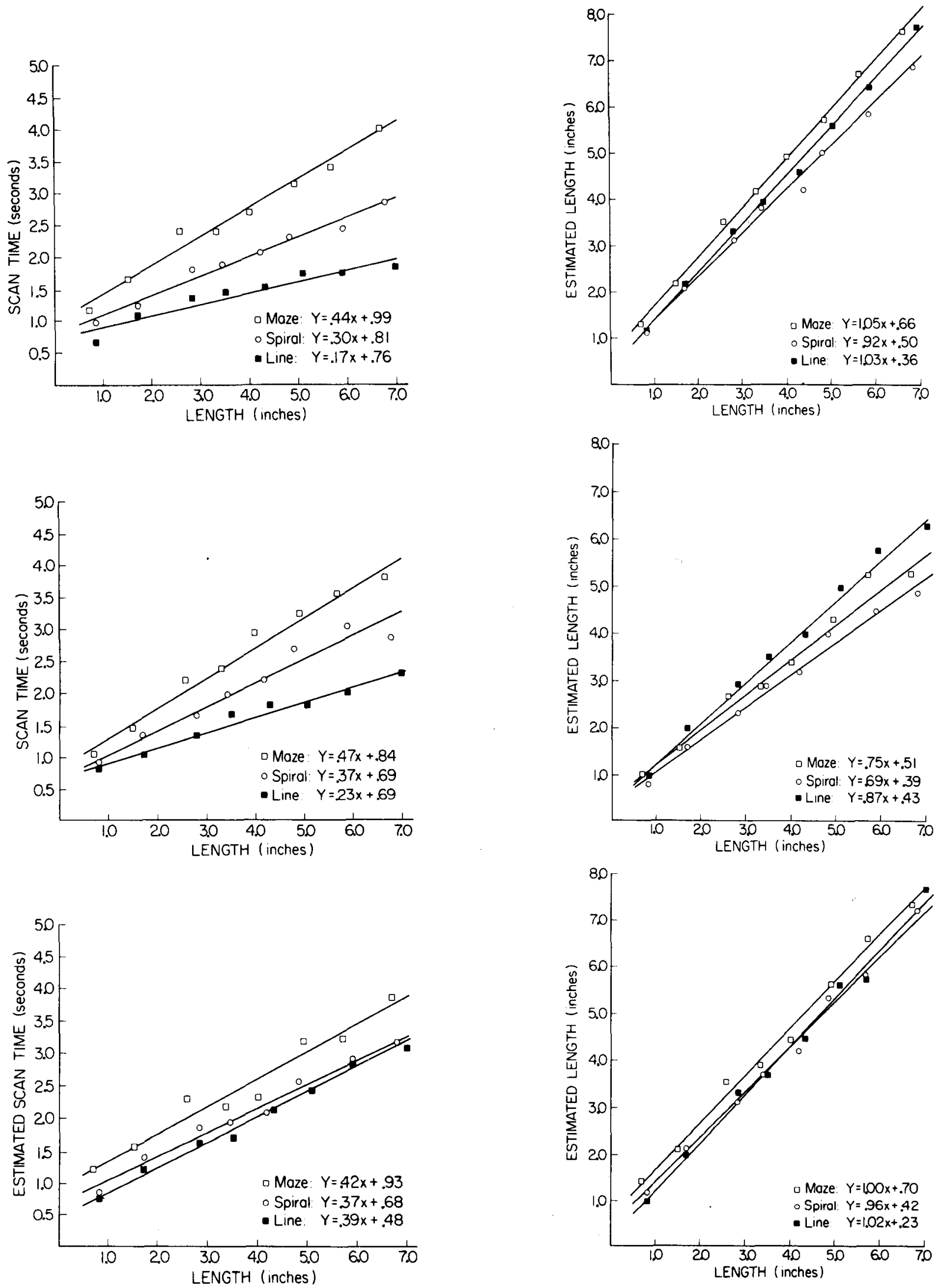

Figure 2. Scanning times and estimated lengths for the perception (top row) and imagery (middle row) conditions of Experiment 1. The bottom row shows estimated scanning times and estimated lengths in Experiment 2. 
Table 1

Correlations and Regression Parameters for Scan Time and Length Estimates

\begin{tabular}{|c|c|c|c|c|c|c|c|c|c|c|}
\hline \multicolumn{3}{|c|}{ Task } & \multicolumn{4}{|c|}{ Scan Time } & \multicolumn{4}{|c|}{ Length Estimate } \\
\hline \multirow{2}{*}{$\begin{array}{c}\text { Experi- } \\
\text { ment }\end{array}$} & \multirow[b]{2}{*}{ Condition } & \multirow[b]{2}{*}{ Stimulus } & \multicolumn{2}{|c|}{$\mathbf{r}$} & \multirow[b]{2}{*}{ Slope } & \multirow[b]{2}{*}{ Intercept } & \multicolumn{2}{|c|}{$\mathbf{r}$} & \multirow[b]{2}{*}{ Slope } & \multirow[b]{2}{*}{ Intercept } \\
\hline & & & G & I & & & $\mathrm{G}$ & I & & \\
\hline 1 & Perception & Line & .95 & .86 & .17 & .76 & 1.00 & .99 & 1.03 & .36 \\
\hline 1 & Perception & Spiral & .99 & .93 & .30 & .81 & 1.00 & .97 & .92 & .50 \\
\hline 1 & Perception & Maze & .99 & .94 & .44 & .99 & 1.00 & .98 & 1.05 & .66 \\
\hline 1 & Imagery & Line & .99 & .81 & .23 & .69 & 1.00 & .98 & .87 & .43 \\
\hline 1 & Imagery & Spiral & .97 & .91 & .37 & .69 & .99 & .97 & .69 & .39 \\
\hline 1 & Imagery & Maze & .99 & .94 & .47 & .84 & 1.00 & .96 & .75 & .51 \\
\hline $1 \mathrm{~A}$ & Perception & Line & .99 & .89 & .26 & .73 & 1.00 & .98 & 1.13 & .21 \\
\hline $1 \mathrm{~A}$ & Perception & Spiral & .97 & .94 & .34 & 1.01 & .99 & .97 & .86 & .66 \\
\hline $1 \mathrm{~A}$ & Perception & Maze & .99 & .96 & .52 & 1.17 & 1.00 & .98 & 1.06 & .48 \\
\hline 2 & Imagery & Line & 1.00 & .80 & .39 & .48 & .99 & .95 & 1.02 & .23 \\
\hline 2 & Imagery & Spiral & .99 & .77 & .37 & .68 & .99 & .97 & .96 & .42 \\
\hline 2 & Imagery & Maze & .98 & .82 & .42 & .93 & 1.00 & .97 & 1.00 & .70 \\
\hline
\end{tabular}

Note $-G=$ group,$I=$ individual .

procedure was identical to the procedure followed by the perception group in Experiment 1 and produced the same pattern of results, we have summarized these results without reporting them in detail.

The combined results demonstrate that the configuration of the line being scanned influences scanning time, and it appears that these configurations have little influence on length estimation. A tacit knowledge hypothesis based only on time and extent cannot account for these results, since perceived length does not predict scanning time across configurations. For that hypothesis to be supported, it would be necessary for people to know how variations in the pattern of the lines influence their scan time. This is considered in Experiment 2 .

\section{EXPERIMENT 2}

A consistent finding in Experiment 1 was that pattern configuration influenced scanning rate. Subjects scanned diagonal lines significantly faster than spirals and spirals significantly faster than mazes. In order for the tacit knowledge hypothesis to account for these results, people would have to anticipate how pattern configuration would influence scan time. Although people can anticipate that scan time increases with linear distance (Mitchell \& Richman, 1980), it is not clear whether they can anticipate the differential configuration effect. The purpose of Experiment 2 was to evaluate this by asking them to estimate scan time for the 24 patterns used in Experiment 1. ${ }^{2}$

\section{Method}

Subjects. The subjects were 12 undergraduates at Florida Atlantic University who were paid $\$ 3$ for their participation.

Procedure. The procedure was identical to that used in the image condition of Experiment 1 for both the length estimation and scanning tasks, except that subjects estimated scan time during the "scanning" task. The near identity of the two procedures was enhanced by initially giving subjects the same instructions used in the image condition of Experiment 1. After they read the instructions, subjects were given the following modified instructions for the scanning task: "The instructions that you read were used in a previous experiment. In this experiment we want you to estimate how long you would take to scan an image without actually scanning it. After the pattern goes off the screen, estimate how long it would take you to scan the image if you attempted to follow the previous instructions. You may make your estimate to the nearest tenth of a second if you wish. You will have nine practice trials before we begin."

\section{Results}

The bottom right panel in Figure 2 shows the relation between the actual length and the estimated length. The correlation between the two variables was .99 for the line, .99 for the spiral, and 1.00 for the maze. The means of the individual correlations were .95 for the line, .97 for the spiral, and .97 for the maze. The slope of the regression line was 1.02 for the line, .96 for the spiral, and 1.00 for the maze. The slopes indicate that the average length judgments were very accurate, in contrast to the underestimation of length found for the imagery condition in Experiment 1. The differences in slope across patterns were not significant $[F(2,22)<1]$.

The bottom left panel in Figure 2 shows that estimated scan time increased with length, as was also reported by Mitchell and Richman (1980). The group correlations were 1.00 for the line, .99 for the spiral, and .98 for the maze. The average individual correlations were .80 for the line, .77 for the spiral, and .82 for the maze.

Unlike the scanning results, these estimated scan rates did not differ significantly across patterns $[F(2,22)<1]$. The estimated scan rate was $.39 \mathrm{sec} / \mathrm{in}$. for the line, $.37 \mathrm{sec} / \mathrm{in}$. for the spiral, and $.42 \mathrm{sec} / \mathrm{in}$. for the maze. However, there was a significant difference in the $y$-intercepts $[F(2,22)=5.18, p<.05]$. The intercept 
values of $.48 \mathrm{sec}$ for the line, $.68 \mathrm{sec}$ for the spiral, and $.93 \mathrm{sec}$ for the maze may reflect that people scan the line more quickly than the spiral and the spiral more quickly than the maze. People may have tacit knowledge about the relative time it takes to scan different configurations, which is reflected by an additive constant (y-intercept) rather than by scan rates (slope differences). These differences among the y-intercepts generally correspond to those found in Experiment 1 . The values for the line, spiral, and maze were $.76, .81$, and .99 for the perception condition and $.69, .69$, and .84 for the image condition. Those times were not significantly different $[F(2,44)=2.77, p<.08]$, but the pattern of the findings and significantly different values of $.73,1.01$, and 1.17 in Experiment $1 \mathrm{~A}[\mathrm{~F}(2,22)=9.67$, $\mathrm{p}<.01$ ] may suggest a response bias due to tacit knowledge, in addition to the actual time it takes to mentally scan a pattern.

\section{DISCUSSION}

The results of these studies can best be accounted for by assuming that length estimation and visual scanning are separate and independent tasks. We did not find any support either for the hypothesis that length estimates are used to simulate mental scanning or for the hypothesis that scanning time is used to estimate lengths. The results are most consistent with the hypothesis that people actually scan in the mental scanning task and do not rely on scanning time to estimate lengths. We consider the length estimation task first and then the scanning task.

\section{Length Estimation}

The argument that scanning time did not influence length estimates is supported by the lack of relationship between the two variables. There were large and consistent differences in scanning times across the three configurations. Scanning was fastest for the line, intermediate for the spiral, and slowest for the maze. Thus the scanning time hypothesis should predict that the line would be judged the shortest and the maze the longest. Instead, the spiral was always judged the shortest and the line was judged similar to the maze.

Another argument against the scanning time hypothesis is that the correlations between length estimates and length are higher than the correlations between scanning time and length. This is particularly evident for the diagonal line. The average individual correlations between scan time and length were .86 and .81 in the two conditions of Experiment 1. The corresponding correlations between estimated length and length were .99 and .98 . The correlations raise the question for the scanning time hypothesis of how a less consistent judgment (scan time) can serve as the basis for a more consistent judgment (estimated length).

In order to give subjects the opportunity to report on their strategies, we designed a questionnaire in which we asked subjects to rate how often they used each of several alternative strategies. The ratings were 0 (not at all), 1 (seldom), 2 (about half of the time), 3 (most of the time), and 4 (all of the time). The ratings of the 24 subjects tested in the perception condition (Experiments 1 and 1A) and the 24 subjects tested in the imagery condition (Experiments 1 and 2) suggest that people usually estimate line length by moving a unit of measurement along the line. The average ratings for this strategy were 2.7 in the perception condition and 1.7 in the imagery condition. The average ratings for using scan time to estimate length were .3 in the perception condition and .7 in the imagery condition.

The strategy of estimating length by moving a standard along a line has also received some support in previous investigations. Hartley (1981) argued that subjects used this strategy because the time it took them to estimate length increased linearly with length. However, this finding is also consistent with the scanning time hypothesis. Thorndyke (1981) proposed that subjects use scanning time to estimate length, but his subjects reported that they estimated length by determining how many times a standard would fit a line and multiplying by the length of the standard. Although Thorndyke argues that these reports are consistent with his timing model, it is not clear why subjects would base their estimates on scanning time if they computed the answer by superimposing a unit of measurement.

Perhaps subjects superimpose a standard but also have knowledge of how long it takes them to complete this procedure. They may then calculate the answer based on this strategy but slightly modify their answer according to how long it took them to calculate it. This time bias would have to be task specific, because it did not occur in our experiments. Another possibility is that Thorndyke's (1981) results are unrelated to scanning time. Further research, including the measurement of scanning time in his paradigm, is necessary to resolve this issue.

\section{Mental Scanning}

Support for the suggestion that tacit knowledge had very little influence on the outcome of the scanning experiments comes from the finding that predicted scanning times are different from the times produced when subjects are instructed to scan the configurations. Although people correctly predict the effect of length on scanning time, they did not correctly predict the effect of configuration on scanning time. Any tacit knowledge that configuration influences scan time was instead represented by differences in the y-intercepts of the regression lines. Subjects correctly predicted that scanning a maze should take longer than scanning a spiral and scanning a spiràl should take longer than scanning a line, but estimates differed by an additive constant that was independent of length. In contrast, when subjects are asked to actually scan patterns, their responses reveal differences in the rate of scanning across configurations, as measured by significant differences in the slopes of the regression lines. These slopes did not 
differ significantly when subjects were asked to estimate scanning time.

A secondary finding that may be difficult to explain in terms of tacit knowledge is how pattern configuration influences the consistency of scanning times. The average individual correlation between scanning time and length was always less for the lines than it was for the spiral and maze (Table 1). It is difficult to foresee how the tacit knowledge hypothesis could account for this finding unless people were also less consistent in esti. mating the lengths of lines than in estimating the lengths of spirals and mazes. This, however, did not occur.

Although the tacit knowledge hypothesis appears to be an inadequate explanation of how people scan complex configurations, it is possible that people might rely on their tacit knowledge to avoid scanning linear distances. However, Finke and Pinker (1982) recently obtained evidence that suggests people mentally scan linear distances even when they were not instructed to scan a mental image. Their paradigm required subjects to judge whether an arrow at an unexpected location pointed at any dots in a previously exposed dot pattern. Reaction times were directly proportional to the distance separating the dots and the arrows, suggesting that the subjects used mental scanning to make inferences about spatial relations.

Our argument that the tacit knowledge hypothesis cannot account for our results supports the more general argument made by Finke (1980) that it is unlikely that people could anticipate the results of many imagery experiments. Finke cites many specific examples in which he claims that the outcome is not obvious, usually because people are doing the task for the first time and their tacit knowledge is incorrect or incomplete. Consistent with that argument, it appears from the data reported here that tacit knowledge is inadequate to account for all mental scanning data.

\section{REFERENCES}

Finke, R. A. Levels of equivalence in imagery and perception. Psychological Review, 1980, 87, 113-132.
Finke, R. A., \& Pinker, S. Spontaneous imagery scanning in mental extrapolation. Journal of Experimental Psychology: Learning, Memory, and Cognition, 1982, 8, 142-147.

Hartley, A. A. Mental measurement of line length: The role of the standard. Journal of Experimental Psychology: Human Perception and Performance, 1981, 7, 309-317.

IntONS-Peterson, M. J. Imagery paradigms: How vulnerable are they to experimenters' expectations? Journal of Experimental Psychology: Human Perception and Performance, in press.

Kerst, S. M., \& Howand, J. H. Memory psychophysics for visual length and area. Memory \& Cognition, 1978, 6, 327-333.

Kosslyn, S. M., Ball, T. M., \& Reiser, B. J. Visual images preserve metric spatial information: Evidence from studies of image scanning. Journal of Experimental Psychology: Human Perception and Performance, 1978, 1, 47-60.

Kosslyn, S. M., \& Pomerantz, J. R. Imagery, propositions, and the form of internal representations. Cognitive Psychology, $1977,9,52-76$.

Mitchell, D. B., \& Richman, C. L. Confirmed reservations: Mental travel. Journal of Experimental Psychology: Human Perception and Performance, 1980, 6, 58-66.

Moyer, R. S., Bradley, D. R., Sorengen, M. H., Whitina, J. C., \& Mansfierd, D. P. Psychophysical functions for perceived and remembered size. Science, 1978, 200, 330-332.

Pylyshyn, Z. W. The imagery debate: Analogue media versus tacit knowledge. Psychological Review, 1981, 88, 16-45.

Thorndyke, P. W. Distance estimation from cognitive maps. Cognitive Psychology, 1981, 13, 526-550.

\section{NOTES}

1. Although the results of Experiment 1 and Moyer et al. (1978) indicate that length is underestimated in the imagery condition, other findings differ. Kerst and Howard (1978) found that estimated distance was slightly greater for the imagery condition. In addition, our results from the imagery condition in Experiment 2 were very similar to the length estimates obtained from the perception condition in Experiment 1.

2. The subjects were tested by an undergraduate who was unfamiliar with previous work on visual imagery and was unaware of the hypotheses that motivated this research. She did not see the results of the experiments until the entire project was completed. We therefore avoided the experimenter-bias effects described by Intons-Peterson (in press).

(Received for publication June 21, 1982; revision accepted September 27, 1982.) 